\title{
Inventaire Et Diversité Des Essences Commerciales \\ Dans Deux Forêts Périurbaines De Brazzaville : Le Site Continental De La Djoumouna Et Le Site Insulaire Loufézou (République Du Congo)
}

\author{
Edmond Sylvestre Miabangana, PhD \\ Herbier National du Congo (IEC), Institut National de Recherche en \\ Sciences Exactes et Naturelles (IRSEN), Cité Scientifique de Brazzaville, \\ République du Congo \\ Agence Nationale de Valorisation des Résultats de la Recherche et de \\ l'Innovation (ANVRI), Cité Scientifique de Brazzaville, \\ République du Congo \\ Eminence Dorelle Hondjuila Miokono, PhD \\ Ecole Nationale Supérieure Polytechnique (ENSP), Université Marien \\ Ngouabi, Brazzaville, République du Congo \\ Gilbert Nsongola, Master étudiant \\ Odile Clarisse Saminou Nkouka, Master \\ Herbier National du Congo (IEC), Institut National de Recherche en \\ Sciences Exactes et Naturelles (IRSEN), Cité Scientifique de Brazzaville, \\ République du Congo

\section{Dieudonné Sita, Ingénieur} \\ Direction des Forêts, Direction Général de l'Economie Forestière, \\ Brazzaville, République du Congo
}

Doi:10.19044/esj.2020.v16n27p217 URL:http://dx.doi.org/10.19044/esj.2020.v16n27p217

Résumé

Un inventaire des essences forestières de bois commercial a été entrepris dans deux forêts périurbaines de Brazzaville : le site continental de la Djoumouna et le site insulaire Loufézou. Ce dernier a été renforcé par le dépouillement des données phytosociologiques et structuraux de végétation. Ces deux habitats réalisent respectivement une surface basale de 35,7 et 53 $\mathrm{m}^{2} /$ ha. Le fond floristique, dominé par les Fabaceae, projette une richesse de 51 espèces émanant respectivement de 462 et 414 individus de $\mathrm{Dbh} \geq 10 \mathrm{~cm}$. Les Pentaclethra eetveldeana (De Wild. et T. Durand) et Pentaclethra macrophylla Benth., dominent l'ossature structurale dans la forêt continentale, tant en surface terrière qu'en indice de valeur d'importance. Sur le site 
insulaire, ce sont Antiaris toxicaria Lesch var. welwitschii (Engl.) Berg et Ceiba pentandra (L.) Gaertn., d'une part, et Pterocarpus soyauxii Taub. et Pentaclethra eetveldeana De Wild. d'autre part qui se relaient pour les deux paramètres. Le site continental est plus riche et plus diversifié par rapport à son voisin insulaire. Tout de même, ces deux habitats particuliers projettent une bonne structuration en essences commerciales, en rapport avec la maturité écologique de ces sites forestiers. Ce trait milite en faveur de l'élaboration des plans d'aménagement à titre conservatoire.

Mots-clés: Essences Commerciales, Diversité Floristique, Aménagement Forestier, Djoumouna, Île Loufézou 


\title{
Inventory And Diversity Of Timber Species In Two Peri-Urban Brazzaville Forests: The Djoumouna Continental Site And The Loufézou Island Site (Republic Of Congo)
}

\author{
Edmond Sylvestre Miabangana, PhD \\ Herbier National du Congo (IEC), Institut National de Recherche en \\ Sciences Exactes et Naturelles (IRSEN), Cité Scientifique de Brazzaville, \\ République du Congo \\ Agence Nationale de Valorisation des Résultats de la Recherche et de \\ l'Innovation (ANVRI), Cité Scientifique de Brazzaville, \\ République du Congo

\section{Eminence Dorelle Hondjuila Miokono, PhD}

Ecole Nationale Supérieure Polytechnique (ENSP), Université Marien Ngouabi, Brazzaville, République du Congo

\section{Gilbert Nsongola, Master étudiant Odile Clarisse Saminou Nkouka, Master}

Herbier National du Congo (IEC), Institut National de Recherche en Sciences Exactes et Naturelles (IRSEN), Cité Scientifique de Brazzaville,

République du Congo

\section{Dieudonné Sita, Ingénieur}

Direction des Forêts, Direction Général de l'Economie Forestière,

Brazzaville, République du Congo

\begin{abstract}
An inventory of commercial timber species was undertaken in two peri-urban forests of Brazzaville, the continental site of Djoumouna and the Loufézou island site. This inventory was strengthened by the analysis of phytosociological and structural vegetation data. These two habitats have a basal area of 35.7 and $53 \mathrm{~m}^{2} /$ ha, respectively. The floristic matrix, dominated by Fabaceae, projects a richness of 51 species emanating respectively from 462 and 414 individuals of Ddh $\geq 10 \mathrm{~cm}$. Pentaclethra eetveldeana De Wild. $\&$ T. Durand and Pentaclethra macrophylla Benth., dominated the structural framework in the continental forest, both in the basal area and the significance of value index. On the island site, Antiaris toxicaria Lesch var. welwitschii (Engl.) Berg and Ceiba pentandra (L.) Gaertn., on the one hand, and Pterocarpus soyauxii Taub. and Pentaclethra eetveldeana De Wild., on the other hand, take turns for both parameters. The continental site is richer and
\end{abstract}


more diversified compared to its island neighbor. All the same, these two particular habitats project a good structure in commercial timber in relation to the ecological maturity of these forest sites. This feature militates in favour of the elaboration of management plans as a precautionary measure.

Keywords: Timber Species, Floristic Diversity, Forest Management, Djoumouna, Loufézou Island

\section{Introduction}

La dégradation des portions d'écosystèmes forestiers, urbains et périurbains, demeure l'une des préoccupations environnementales auxquelles sont confrontés les pays d'Afrique centrale (Marien, 2008). Cette sous-région abrite le bassin du Congo, la deuxième plus grande étendue de forêt tropicale humide dans le monde, avec une superficie de 2 millions de $\mathrm{km}^{2}$ (Mayaux et al., 1998). Ce dernier est au centre des préoccupations internationales sur le changement climatique et pour la sauvegarde de sa biodiversité.

Quant à la périphérie de ce bassin, en raison de la croissance démographique et du développement économique, on note une matrice paysagère fortement anthropisée. Conjuguée aux aléas paléoclimatiques, ce trait justifie l'écorégion de mosaïques forêts-savanes du Congo-Ogooué (White \& Vande Weghe, 2008) à laquelle est intégré le Plateau des Cataractes.

Dans cette entité physiographique, de nombreuses forêts matures ont été écrémées pour l'occupation et l'utilisation des sols. Cependant, quelques rares îlots forestiers, épargnés de l'emprise humaine, le sont, soit en raison d'une protection privée, telle que le cordon ripicole continental de la Djoumouna, soit en raison de leur isolement naturel au sein des domaines insulaires du fleuve Congo, comme l'île forestière Loufézou. En outre, ces deux sites forestiers font partie des sites Ramsar des Rapides du Congo-Djoué (Anonyme, 2009).

Ces deux portions écosystémiques ont fait l'objet d'investigations écologiques, au cours de ces dix dernières années. En ce qui concerne l'îlot forestier continental de la Djoumouna, ces travaux ont été orientées sur l'analyse floristique et phytogéographique (Miabangana et al., 2016), la caractérisation phytosociologique (Miabangana, 2019) et structurale (Miabangana, 2020). Quant au site insulaire, des travaux similaires y sont également répertoriés. Ils font référence aux études floristiques et phytogéographiques (Miabangana, 2012 ; Miabangana \& Lubini, 2015), phytosociologiques (Miabagana, 2019) et structurales (Miabangana \& Malaisse, 2020).

En ce qui concerne les services écosystémiques y afférents, ils se déclinent en services d'approvisionnement pour les populations riveraines. Les premiers inventaires ethnobotaniques ont couvert le site de la Djoumouna 
(Miabangana, 1998). Ils se sont focalisés sur l'état des lieux des plantes utiles de ce site. Par la suite, des inventaires approfondis se sont focalisés sur les plantes à usage alimentaire (Miabangana \& Hondjuila-Miokono, 2015) et les plantes médicinales (Miabangana \& Hondjuila-Miokono, 2016).

Outre les services d'approvisionnement sus-repris, et malgré les faibles superficies, ces deux sites affichent une richesse relative en essences commerciales, comparativement à d'autres lambeaux dégradés. Des investigations ciblées et complétées par le dépouillement des données phytosociologiques et structurales, déjà mentionnées, confortent leur diversité relative dans ces deux sites forestiers.

Dans ce sens, cette note se propose l'inventaire et la distribution des essences commerciales des deux forêts suburbaines, en vue d'affiner la connaissance de leur phytodiversité à des fins d'une gestion durable.

\section{Méthodes d'étude}

Sites d'étude

Les deux sites forestiers prospectés sont localisés dans le Plateau des Cataractes, au sud-est de Brazzaville (Figure 1). L'îlot forestier de la Djoumouna, localisé dans la fraction continentale (longitudes E1509'18''E15 $08^{\prime} 42^{\prime \prime}$ et les latitudes $\mathrm{S} 4^{\circ} 21^{\prime} 18^{\prime \prime}$ 'S $\left.4^{\circ} 21^{\prime} 54^{\prime \prime}\right)$. Il est situé à $24 \mathrm{~km}$ de Brazzaville, dans la Sous-Préfecture de Goma tsé-tsé, dans le Département du Pool. D'une longueur maximale de 620 mètres et d'une largeur correspondante de 270 mètres, la forêt couvre une superficie de 10,2 hectares.

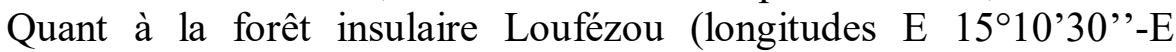
$15^{\circ} 09^{\prime} 54^{\prime \prime}$ et

latitudes $\left.4^{\circ} 22^{\prime} 11.65^{\prime} \mathrm{S}-4^{\circ} 22^{\prime} 47.91 \mathrm{~S}\right)$, une des quatre îles forestières du fleuve Congo dans le Plateau des Cataractes, elle couvre une superficie de 50 hectares. Elle est située à l'interface entre la Sous-Préfecture sus-reprise et le Département de Brazzaville. Le gradient altitudinal va de $245 \mathrm{~m}$ des rebords du fleuve pendant la période d'étiage maximale lors de la saison sèche, à 290 $\mathrm{m}$ sur le plateau de l'île. D'une longueur maximale de 875 mètres et d'une largeur correspondante de 500 mètres, l'île couvre une superficie de 56,3 hectares. 


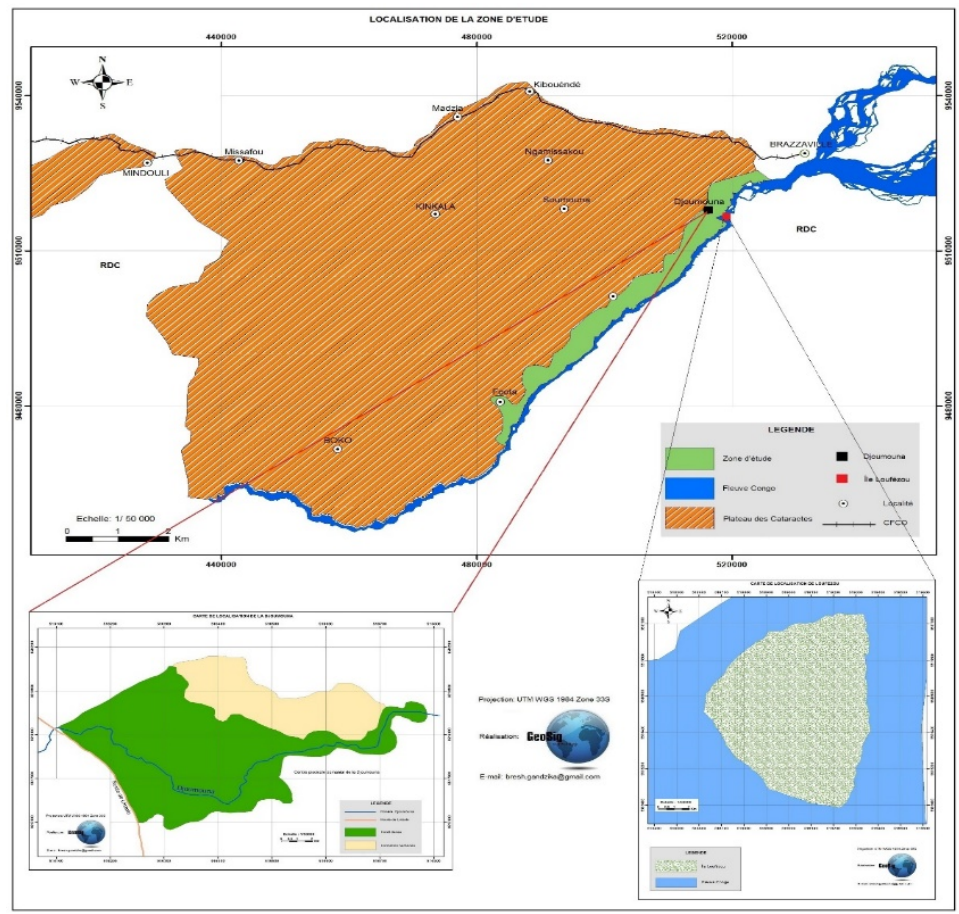

Figure 1. Localisation des deux sites forestiers prospectés

Le climat est tropical humide, de type bas-congolais (Samba-Kimbata, 1978 ; Atlas, 2001) qui, selon la classification de Köppen (1936), est de type AW4 ; c'est-à-dire, caractérisé par une saison pluvieuse longue, entrecoupée par une saison sèche de trois à quatre mois, de juin à septembre.

Le substratum géologique est représenté essentiellement par des formations sédimentaires du Précambrien supérieur, de nature schistogréseuse où elles forment la série de l'Inkisi, et composé des arkoses et des grès feldspathiques, alors que les sols appartiennent à la classe des sols ferralitiques fortement désaturés remaniés jaunes (Denis, 1974).

$\mathrm{Au}$ niveau de la chorologie locale, les deux sites prospectés sont situés dans un écotone phytogéographique entre les sous-centres Congolais et BasGuinéen (Miabangana, 2019), dans le secteur de transition bas-guinéozambézien, à l'échelle paysagère et du District floristique du Plateau des Cataractes (Kimpouni et al., 1992).

\section{Méthodes de collecte et de traitement des données}

Les premières investigations ethnobotaniques ont commencé en 1998 dans la forêt continentale de la Djoumouna. Elles se focalisaient en un inventaire des plantes utiles du site, au nombre desquelles figuraient en bonne place les essences commerciales. 
De 2013 à 2020, des prospections complémentaires, axées sur des études phytosociologiques et structurales, ont été entreprises concomitamment sur la forêt continentale de la Djoumouna et sur la forêt insulaire de Loufézou.

À partir des relevés polyvalents de $625 \mathrm{~m}^{2}$ chacune, toutes les espèces vasculaires ont été prises en compte pour les relevés phytosociologiques. Quant aux relevés structuraux, seuls les arbres et les arbustes réalisant une circonférence à hauteur de poitrine, $\mathrm{CHP} \geq 31,4 \mathrm{~cm}$ (équivalent du diamètre à hauteur de poitrine, DHP $\geq 10 \mathrm{~cm}$ ou DBH : Diameter at breast height, pour les anglo-saxons), ont été soumis à des mesures dendrométriques. Dans les relevés structuraux, les individus hors relevé ont été également soumis à des mensurations et intégrés au relevé adjacent (White \& Edwards, 2000). Des ajustements préconisés par Pierlot (1966) ont été pris en compte. Ces derniers concernent, dans la présente étude, des individus présentant des racines échasses, des contreforts et des cannelures. 25 et 33 relevés ont été exécutés respectivement dans la forêt continentale de la Djoumouna et dans la forêt insulaire Loufézou.

Le dépouillement des données y afférentes ont permis de compléter la liste originelle des essences commerciales de la forêt de la Djoumouna. Il a, en outre, permis l'élaboration d'une cartographie fiable de ces essences dans les deux types forestiers.

L'identification des espèces, amorcée sur le terrain, a été complétée et confirmée à l'Herbier National du Congo (IEC). Des ouvrages fort bien illustrés (Tailfer, 1990 ; Hawthorne \& Jongkind, 2006 ; Hawthorne \& Gyakary, 2006 ; Harris \& Wortley, 2008 ; Meunier et al., 2015 ; Vande weghe et al., 2016), ont été consultés pour une détermination formelle. En ce qui concerne les essences commerciales, quelques travaux (Louppe et al., 2008 ; Vivien \& Faure, 2011; Mille \& Louppe, 2015; ATIBT, 2016; Martin \& Vernay, 2016) ont également affiné l'identification et renseigné sur leur transposition en noms pilotes.

La nomenclature adoptée suit l'APG IV (2016), renforcée par le référentiel taxonomique de Lebrun et Stork (1991-2015) et accessible sur le site régulièrement mis à jour.

\section{Analyse des données}

\section{Paramètres de caractérisation botanique}

Ces derniers concernent la diversité des familles (DiF), la surface terrière relative $\left(\mathrm{St}_{\mathrm{r}}\right)$, la densité relative $\left(\mathrm{D}_{\mathrm{r}}\right)$, la dominance relative $\left(\mathrm{Do}_{\mathrm{r}}\right)$, la fréquence relative $\left(\mathrm{F}_{\mathrm{r}}\right)$ et à l'indice de valeur d'importance relative $\left(\mathrm{IVI}_{\mathrm{r}}\right)$.

La diversité des familles $(\mathrm{DiF})$

$$
\text { DiF }=\frac{\text { Nombre d'espèces de la famille }}{\text { Nombre total d'espèces }} \times 100 \text { (1) }
$$

La surface terrière relative $(\mathrm{Str})$ 


$$
S t r=\sum_{i=1}^{n} \frac{\pi D i 2}{4}
$$

Str : surface terrière relative, exprimée en $\mathrm{m}^{2} / \mathrm{ha} ; D i:$ diamètre à $1,3 \mathrm{~m}$ du sol de l'arbre $i ; n$ : nombre total d'individus de l'espèce. La surface terrière totale (Stt) correspond à la somme des surfaces terrières de tous les individus de la composition floristique, présents sur la surface inventoriée.

La densité relative (Dr)

$$
D r=\frac{\text { Nombre d'individus de l'espèce }}{\text { Nombre total d'individus de toutes les espèces }} \times 100
$$

La dominance relative (Dor)

$$
\text { Dor }=\frac{\text { Surface terrière de liespèce }}{\text { Somme des surfaces terrières de toutes les espèces }} \times 100
$$

La fréquence relative (Fr)

$$
F r=\frac{\text { Fréquence d'une espèce }}{\text { Somme des fréquences de toutes les espèces }} \times 100 \text { (5) }
$$

La fréquence d'une espèce correspond au nombre d'occurrences contenant cette espèce dans une unité d'échantillonnage.

L'indice de valeur d'importance relatif (IVIr)

$$
I V I r=D r+D o r+F r(6)
$$

Ce dernier indice permet de déterminer les espèces les plus dominantes dans l'aire d'échantillonnage. Il est dérivé de l'Importance Value Index de Curtis et McIntosh (1951) et repris par quelques auteurs en Afrique Équatoriale Atlantique (Reitsma, 1988 ; Lejoly, 1993 ; Doucet, 2003). Ainsi, les espèces les plus dominantes (Leading dominant) sont celles qui réalisent les plus grandes valeurs d'IVIr.

\section{Les indices de diversité}

Quelques indices de diversité ont été calculés :

\section{La richesse et la diversité floristiques}

La richesse floristique $(\mathrm{S})$ est le nombre total d'espèces rencontrées dans un peuplement. Quant à la diversité, elle renvoie à la répartition de l'effectif total $(\mathrm{N})$ entre les différentes espèces.

L'indice de Shannon et Weaver (H')

$$
H^{\prime}=-\sum P i l n P i
$$

où $P i$ est l'abondance relative de l'espèce $i(\mathrm{Pi}=\mathrm{ni} / \mathrm{N}), n i:$ nombre d'individus par espèce, $N$ : nombre d'individus total par unité d'échantillonnage. $\mathrm{H} 1$ varie de 0 à 5 bits, voire un peu plus de 5 . Une valeur élevée de cet indice traduit des conditions stationnelles favorables à l'installation de 
nombreuses (espèces)?. Ce trait exprime une grande stabilité du milieu (Dajoz, 1996).

L'indice de Simpson

$$
D=\sum(P i)^{2}
$$

L'indice de Simpson représente la probabilité pour que deux individus pris $\mathrm{au}$ hasard dans le peuplement étudié appartiennent à la même espèce. Il mesure la manière avec laquelle les individus se répartissent entre les espèces d'une communauté. Sa valeur tend vers 0 pour indiquer le maximum de diversité et vers 1 pour indiquer le minimum de diversité.

L'indice de Margalef $\left(R_{M g}\right)$

$$
R_{M g}=\frac{S-1}{\operatorname{Ln}(N)}
$$

d'une

La valeur de l'indice de Margalef renseigne si la richesse spécifique communauté végétale est élevée ou non. Il permet de comparer la richesse floristique entre les deux portions d'écosystèmes prospectées. L'équitabilité de Piélou (1966)

$$
E=\frac{H^{\prime}}{\operatorname{LnS}}=\frac{H^{\prime}}{\operatorname{Ln} S}
$$

La

En ce qui concerne l'équitabilité de Piélou, sa valeur varie de 0 à 1 . élevée est un indicateur d'un peuplement équilibré (Dajoz, Op-cit). Les intervalles des valeurs mentionnées par Inoussa et al. (2013) permettent d'apprécier le degré d'équitabilité d'une phytocénose.

\section{Résultats et discussion}

\section{Caractéristiques floristiques}

Les essences commerciales dans les deux sites, projettent une matrice globale de 51 espèces et taxons infra spécifiques. Ces dernières s'organisent en en 44 genres et 21 familles. La forêt continentale de la Djoumouna présente une richesse floristique de 42 espèces, alors que le site insulaire Loufézou en recèle 25 .

Les familles les plus diversifiées se recrutent parmi les Fabaceae, les Malvaceae, les Myristicaceae, les Moraceae, les Sapotaceae, les Burseraceae et les Anacardiaceae. La Figure 2 reprend les proportions y afférentes des 7 familles les plus représentatives. 


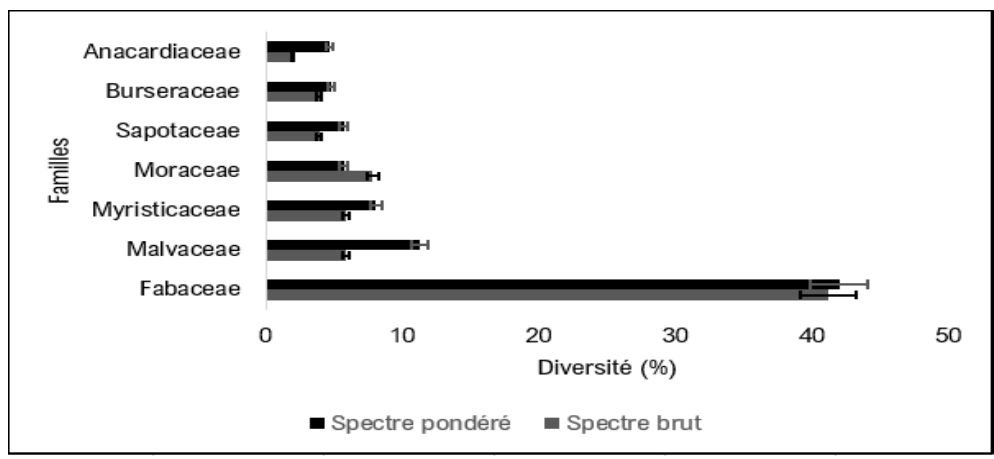

Figure 2. Diversité des familles les plus représentées

Les genres les plus diversifiés sont respectivement Dialium (3 espèces), Amphimas, Celtis, Pentaclethra et Sterculia. Ces quatre derniers renferment tous 2 espèces.

Ces observations reflètent les données de la richesse spécifique globale entre les deux sites prospectés. En effet, la matrice floristique globale de la Djoumouna, uniquement sur terre ferme, renferme 458 espèces (Miabangana et Lubini, 2015). Celle de l'île forestière Loufézou, intégrant le substrat de terre ferme ainsi que le substrat édaphique sur sol hydromorphe, représente 376 espèces (Miabangana et al., 2016). Ce fond floristique dominé par les Fabaceae, avec en tête les Caesalpinoideae (10 espèces sur 18), sousfamille très ancienne, confirme les observations de White (1986), à propos des forêts denses d'Afrique.

Ces deux sites réalisent un indice de connexion floristique de $91 \%$ d'après le coefficient de Sörensen. Ce trait corrobore les observations antérieures (Miabangana, 2019) et conforte l'hypothèse de leur coévolution depuis le Crétacé.

\section{Caractéristiques structurales}

Les individus de $D_{1,3} \geq 10 \mathrm{~cm}$ des forêts de la Djoumouna et de Loufézou réalisent respectivement 35,7 et $53 \mathrm{~m}^{2} /$ ha de surface basale. La liste floristique de tous les individus inventoriés, est reprise au Tableau 1.

En dehors des essences mentionnées au Tableau 1, quelques-unes sont localisées hors de la forêt dense. Sur le site de la Djoumouna, elles occupent, en position résiduaire, des habitats mités par l'action anthropique. Sur substrat de terre ferme, est décelé un pied isolé d'Alstonia boonei De Wild (Emien). Quant au substrat marécageux, quelques individus de faible Dbh, relevant de Mitragyna stipulosa (DC.) Kuntze (Abura), de Symphonia globilifera L. (Ossol, Manil) et Alstonia congensis Engl. (Emien) sont observés. 
Il en est de même de la forêt insulaire, où l'on note quelques individus isolés de Ricinodendron heudelotii (Baill.) Pierre ex Heckel (Essessang), sur terre ferme en voie de reconstitution forestière. Dans sa fraction périodiquement inondée, une abondance de Manilkara obovata (Sabine \& G.Don) J.H.Hemsl. (Monghinza), de Uapaca heudelotii Baill. (Rikio) et de Guibourtia demeusei (Harms) J.Léonard (Paka) est observée. Le bois de ces espèces, atteignant des diamètres exploitables, est sollicité en scierie artisanale sur les rives continentales.

$\mathrm{Au}$ niveau spécifique, Pentaclethra eetveldeana et Pentaclethra macrophylla réalisent les valeurs les plus élevées de surface terrière dans la forêt de la Djoumouna (Figure 3A); alors que Antiaris toxicaria var. welwitschii, Ceiba pentandra, Staudtia kamerunensis var. gabonensis et Pterocarpus soyauxii confortent leur poids dans la forêt insulaire Loufézou (Figure 3B).

Les valeurs exceptionnelles des deux Pentaclethra, en ce qui concerne les surfaces terrières, n'est guère surprenant. En effet, ces essences impriment l'emprise structurale dans cette forêt (Miabangana, 2020), si bien qu'en les considérant sous le prisme d'essences commerciales, elles gardent les mêmes valeurs pondérales. Quant à la forêt insulaire Loufézou, la tendance n'est pas la même. En effet, cette dernière est dominée structuralement par Cynometra pedicellata De Wild. et Dichapetalum barteri Engl. (Miabangana et Malaisse, 2020). Ces deux derniers idiotaxons n'étant pas listés parmi les essences à bois commercial, se trouvent supplantés par d'autres, connus pour leurs propriétés et usages de leurs bois. 
Tableau 1. Liste des paramètres structuraux des espèces (suite et fin)

\begin{tabular}{|c|c|c|c|c|c|c|c|c|c|}
\hline \multirow{2}{*}{$\mathrm{N}^{\circ}$} & \multirow{2}{*}{ Espèces de $D_{1,3} \geq 10 \mathrm{~cm}$ du peuplement forestier } & \multirow{2}{*}{$\begin{array}{c}\text { Famille } \\
\text { Sous-Famille }\end{array}$} & \multirow{2}{*}{ Nom Pilote } & \multicolumn{2}{|c|}{ Densité } & \multicolumn{2}{|c|}{$\mathrm{St}_{\mathrm{rel}}$} & \multicolumn{2}{|c|}{ IVI } \\
\hline & & & & Djoum & Louf & Djoum & Louf & Djoum & Louf \\
\hline 1 & Pentaclethra eetveldeana De Wild. et T.Durand & Fabaceae-Mimosoideae & Mubala & 106 & 64 & 11,4669 & 4,92 & 53,719 & 33,364 \\
\hline 2 & Pentaclethra macrophylla Benth. & Fabaceae-Mimosoideae & Mubala & 66 & 29 & 9,93779 & 2,275 & 41,388 & 19,891 \\
\hline 3 & Pterocarpus soyauxii Taub. & Fabaceae-Faboideae & Padouk & 0 & 52 & 0 & 5,81 & 0 & 33,867 \\
\hline 4 & $\begin{array}{c}\text { Sterculia dawei Sprague } \\
\text { Staudtia kamerunensis Warb. var. gabonensis }\end{array}$ & Malvaceae-Sterculioideae & / & 0 & 42 & 0 & 4,92 & 0 & 32,621 \\
\hline 5 & (Warb.) Fouilloy & Myristicaceae & Niové & 3 & 58 & 0,2585 & 5,892 & 2,8811 & 31,472 \\
\hline 6 & Lannea welwitschii (Hiern.) Engl. & Anacardiaceae & Kumbi & 4 & 43 & 0,35027 & 2,275 & 2,8811 & 25,558 \\
\hline 7 & $\begin{array}{l}\text { Ceiba pentandra (L.) Gaertn. } \\
\text { Antiaris toxicaria Lesch var. welwitschii (Engl.) }\end{array}$ & Malvaceae-Bombacoideae & Fuma, Fromager & 2 & 22 & 0,24531 & 6,674 & 2,8811 & 24,83 \\
\hline 8 & Berg & Moraceae & Ako & 0 & 9 & 0 & 7,804 & 0 & 20,977 \\
\hline 9 & Quassia silvestris Cheek \& Jongkind & Simaroubaceae & Nom Ozek & 29 & 0 & 5,06984 & 0 & 20,472 & 0 \\
\hline 10 & Musanga cecropiö̈des R.Br. & Urticaceae & Parasolier & 24 & 0 & 3,80678 & 0 & 15,271 & 0 \\
\hline 11 & Gilbertiodendron dewevrei (De Wild.) J.Léonard & Fabaceae-Caesalpinioideae & Limbali & 24 & 0 & 2,90034 & 0 & 15,028 & 0 \\
\hline 12 & Chrysophyllum subnudum Baker & Sapotaceae & Longhi & 16 & 13 & 1,7624 & 0,975 & 14,945 & 7,8469 \\
\hline 13 & Maesopsis eminii Engl. & Rhamnaceae & Musizi & 6 & 20 & 0,30953 & 1,729 & 3,9336 & 13,826 \\
\hline 14 & Canarium schweinfurthii Engl. & Burseraceae & Aiélé & 3 & 13 & 0,5181 & 2,526 & 2,9664 & 12,503 \\
\hline 15 & Manilkara aubrevillei Sillans & Sapotaceae & & 19 & 0 & 1,38639 & 0 & 11,686 & 0 \\
\hline 16 & Allanblackia floribunda Oliv. & Clusiaceae & Nsangomo & 25 & 0 & 1,47352 & 0 & 10,37 & 0 \\
\hline 17 & Uapaca guineensis Müll.Arg. & Phyllanthaceae & Rikio & 16 & 1 & 1,92765 & 0,008 & 10,162 & 0,8279 \\
\hline 18 & Celtis tessmannii Rendle & Cannabaceae & $\begin{array}{l}\text { Diania, Celtis } \\
\text { d'Afrique }\end{array}$ & 10 & 0 & 1,05229 & 0 & 10,061 & 0 \\
\hline 19 & Dacryodes pubescens (Vermoesen) H.J.Lam & Burseraceae & Safukala & 3 & 12 & 0,67855 & 1,631 & 3,7167 & 9,4207 \\
\hline 20 & Pycnanthus angolensis (Welw.) Warb & Myristicaceae & Ilomba & 11 & 6 & 1,22656 & 0,696 & 8,281 & 5,0543 \\
\hline 21 & Millettia laurentii De Wild. & Fabaceae-Faboideae & Wengé & 13 & 1 & 1,45947 & 0,132 & 8,2088 & 1,0633 \\
\hline 22 & Sterculia tragacantha Lindl. & Malvaceae-Sterculioideae & Efok Afum & 0 & 10 & 0 & 0,997 & 0 & 7,7356 \\
\hline
\end{tabular}




\begin{tabular}{|c|c|c|c|c|c|c|c|c|c|}
\hline 23 & Petersianthus macrocarpus (P.Beauv.) Liben & Lecythidaceae & Essia & 10 & 0 & 1,2178 & 0 & 6,663 & 0 \\
\hline 24 & $\begin{array}{c}\text { Aphanocalyx microphyllus (Harms) Wieringa subsp. } \\
\text { Microphyllus }\end{array}$ & Fabaceae-Caesalpinioideae & Andoung & 4 & 0 & 0,68295 & 0 & 6,2505 & 0 \\
\hline 25 & Hymenostegia neoaubrevillei J. Léonard & Fabaceae-Caesalpinioideae & / & 7 & 0 & 1,29133 & 0 & 6,146 & 0 \\
\hline 26 & Morus mesozygia Stapf & Moraceae & Difou & 0 & 7 & 0 & 1,294 & 0 & 5,8592 \\
\hline 27 & Scorodophloeus zenkeri Harms & Fabaceae-Caesalpinioideae & Divida & 7 & 0 & 0,77566 & 0 & 5,6809 & 0 \\
\hline 28 & Cynometra sessiliflora Harms & Fabaceae-Caesalpinioideae & / & 5 & 0 & 0,6594 & 0 & 4,5771 & 0 \\
\hline 29 & Treculia africana Decne. & Moraceae & / & 0 & 4 & 0 & 0,259 & 0 & 3,7424 \\
\hline 30 & Irvingia grandifolia (Engl.) Engl. & Irvingiaceae & Andongwé & 2 & 0 & 1,19194 & 0 & 3,4994 & 0 \\
\hline 31 & Dialium corbisieri Staner & Fabaceae-Caesalpinioideae & Éyoum & 4 & 0 & 0,45923 & 0 & 3,077 & 0 \\
\hline 32 & Oddoniodendron romeroi Mendes & Fabaceae-Caesalpinioideae & / & 4 & 0 & 0,21195 & 0 & 2,6325 & 0 \\
\hline 33 & Dialium tessmannii Harms & Fabaceae-Caesalpinioideae & Éyoum & 3 & 0 & 0,58875 & 0 & 2,6315 & 0 \\
\hline 34 & Strombosia pustulata Oliv. & Strombosiaceae & Afina & 9 & 1 & 0,07065 & 0,049 & 2,5369 & 0,906 \\
\hline 35 & Ganophyllum giganteum (A.Chev.) Hauman & Sapindaceae & Mokenjo & 0 & 2 & 0 & 0,439 & 0 & 2,4589 \\
\hline 36 & Erythrophleum suaveolens (Guill. et Perr.) Brenan & Fabaceae-Caesalpinioideae & Tali & 4 & 0 & 0,0584 & 0 & 2,3565 & 0 \\
\hline 37 & Albizia ferruginea (Guill. et Perr.) Benth. & Fabaceae-Mimosoideae & Iatandza & 3 & 0 & 0,65351 & 0 & 2,286 & 0 \\
\hline 38 & Milicia excelsa (Welw.) C.C.Berg & Moraceae & Iroko, Kambala & 3 & 1 & 0,37947 & 0,708 & 1,7934 & 2,1568 \\
\hline 39 & Piptadeniastrum africanum (Hook.f.) Brenan & Fabaceae-Mimosoideae & Dabéma & 2 & 0 & 0,3925 & 0 & 2,0623 & 0 \\
\hline 40 & Berlinia grandiflora (Vahl) Hutch. \& Dalziel & Fabaceae-Caesalpinioideae & & 3 & 0 & 0,11532 & 0 & 1,7804 & 0 \\
\hline 41 & Terminalia superba Engl. \& Diels & Combretaceae & Limba, Fraké & 0 & 1 & 0 & 0,502 & 0 & 1,766 \\
\hline 42 & Dialium pachyphyllum Harms & Fabaceae-Caesalpinioideae & Éyoum & 3 & 0 & 0,05401 & 0 & 1,6702 & 0 \\
\hline 43 & Anopyxis klaineana (Pierre) Engl & Rhizophoraceae & Bodioa & 1 & 0 & 0,33166 & 0 & 1,2746 & 0 \\
\hline 44 & Daniellia klainei (Pierre) De Wild. & Fabaceae-Caesalpinioideae & Faro & 1 & 0 & 0,33166 & 0 & 1,2746 & 0 \\
\hline 45 & Coelocaryon preussii Warb. & Myristicaceae & Ékouné & 0 & 1 & 0 & 0,096 & 0 & 0,9954 \\
\hline 46 & Amphimas pterocarpoides Harms & Fabaceae-Faboideae & Lati & 2 & 0 & 0,02897 & 0 & 0,9469 & 0 \\
\hline 47 & Entandrophragma angolense (Welw.) C.DC. & Meliaceae & Tiama & 1 & 0 & 0,08038 & 0 & 0,8228 & 0 \\
\hline
\end{tabular}




\begin{tabular}{|c|c|c|c|c|c|c|c|c|c|}
\hline 48 & Celtis adolfi-friderici Engl. & Cannabaceae & $\begin{array}{l}\text { Diania, Celtis } \\
\text { d'Afrique }\end{array}$ & 1 & 0 & 0,07065 & 0 & 0,8053 & 0 \\
\hline 49 & Ongokea gore (Hua) Pierre & Aptandraceae & Angueuk & 1 & 0 & 0,06154 & 0 & 0,789 & 0 \\
\hline 50 & Berlinia bruneelii (De Wild.) Torre \& Hillc. & Fabaceae-Caesalpinioideae & Ebiara & 1 & 0 & 0,04906 & 0 & 0,7665 & 0 \\
\hline 51 & Amphimas ferrugineus Pierre ex Pellegr. & Fabaceae-Faboideae & Lati & 1 & 2 & 0,04153 & 0,107 & 0,753 & 1,2584 \\
\hline & & & TOTAL : & 462 & 414 & 53,6383 & 109,3 & 300 & 300 \\
\hline
\end{tabular}

Légende :

0 : absence de l'espèce dans le site ;

Djoum : site forestier continental de la Djoumouna ;

Louf : site forestier insulaire Loufézou 

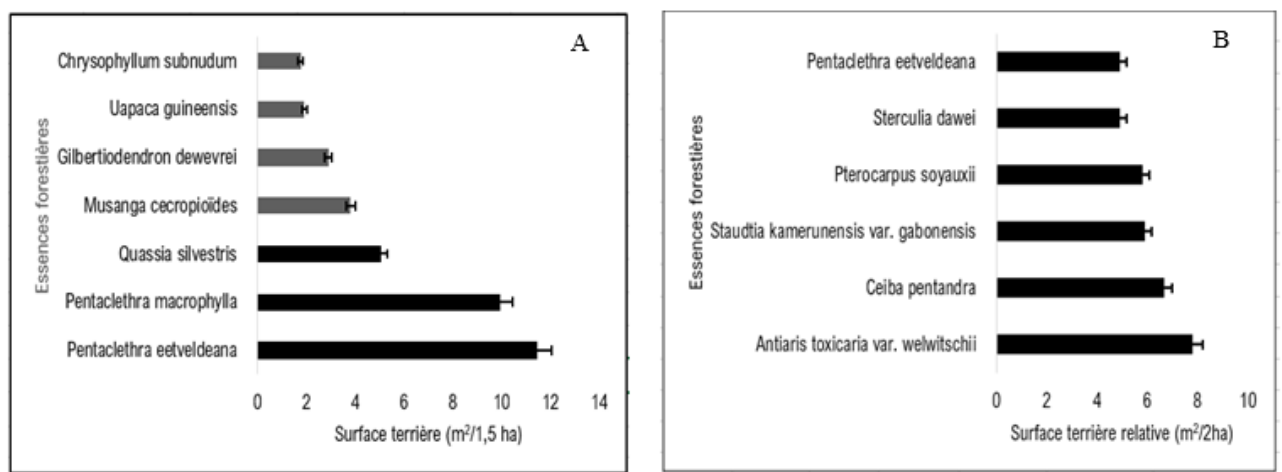

Figure 3. Essences réalisant les valeurs les plus élevées en surface terrière

En ramenant les résultats à l'hectare, les forêts de la Djoumouna et de Loufézou réalisent respectivement 308 et 200 individus par unité d'échantillonnage, valeurs inférieures par rapport à la prévision de Rollet (1983) et de Pascal (2003). En effet, ces auteurs la projettent dans l'intervalle de 450-750 individus. Ce trait se justifie par le fait que dans cet inventaire, seules les essences commerciales ont été prises en compte, parmi tout le potentiel ligneux que regorge ces deux sites forestiers. Ces faits sont confortés par les valeurs obtenues lors des études structurales dans ces deux forêts. 653 individus ont été inventoriés dans la forêt de la Djoumouna (Miabangana, Opcit) et 757 dans le site insulaire Loufézou (Miabangana et Malaisse, Op-cit).

En ce qui concerne les indices d'importance des valeurs, Pentaclethra eetveldeana est l'essence qui projette la valeur la plus élevée dans les deux portions forestières. Dans le site insulaire, elle se partage le leading dominant avec Pterocarpus soyauxii. Cette dernière essence forme un faciès particulier, avec Cynometra pedicellata De Wild., au sein de cet habitat forestier (Miabangana, inédit).

Dans la forêt de la Djoumouna, les valeurs élevées de surface terrière se superposent à celles d'IVI. Pentaclethra eetveldeana et Pentaclethra macrophylla forment l'ossature structurale dans cette forêt continentale (Miabangana, 2020). La Figure 4 présente les 10 espèces aux valeurs élevées, dans la forêt de la Djoumouna (4A) et de Loufézou (4B). 

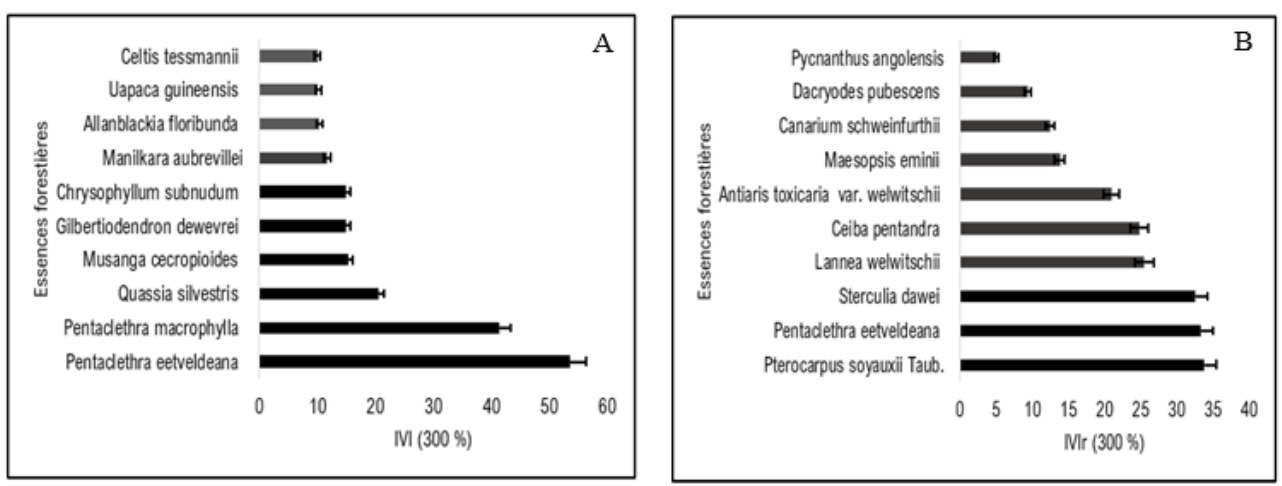

Figure 4. Essences réalisant les valeurs les plus élevées en IVI

La prévision de Reitsma (1988) étant de IVIr $\geq 10$, toutes les essences reprises à la Figure 4A renforcent l'armature structurale des essences commerciales de la forêt de la Djoumouna. Quant à la fraction insulaire, à l'exception de Pycnanthus angolensis, les autres reprises à la Figure 4B participent aussi à une envergure similaire.

En ce qui concerne les indices de diversité, ils sont l'expression dérivée des études structurales entreprises dans ces deux habitats forestiers, la Djoumouna (Miabangana, Op-cit) et Loufézou (Miabangana et Malaisse, Opcit). La fraction continentale de la Djoumouna étant plus riche et diversifiée tant pour la florule totale que sur les essences commerciales. Ce trait est justifié par les différentes valeurs des indices de diversité repris dans le Tableau 2.

Par rapport à l'indice de Shannon-Weaver, sa valeur exceptionnelle élevée dans la forêt de la Djoumouna traduit une bonne diversité des essences commerciales. Cette observation est imputable à sa richesse floristique relativement supérieure à celle de sa voisine insulaire. L'équitabilité de Piélou et l'indice de Margalef suivent la même tendance.

Tableau 2. Comparaison des indices de diversité entre les deux habitats forestiers.

\begin{tabular}{|c|c|c|c|c|}
\cline { 2 - 4 } \multicolumn{1}{c|}{} & \multicolumn{2}{c|}{$\begin{array}{c}\text { Forêt continentale de la } \\
\text { Djoumouna }\end{array}$} & \multicolumn{2}{c|}{$\begin{array}{c}\text { Forêt insulaire } \\
\text { Loufézou }\end{array}$} \\
\cline { 2 - 5 } & $\begin{array}{c}\text { Structure } \\
\text { forestière }\end{array}$ & $\begin{array}{c}\text { Essences } \\
\text { commerciales }\end{array}$ & $\begin{array}{c}\text { Structure } \\
\text { forestière }\end{array}$ & $\begin{array}{c}\text { Essences } \\
\text { commerciales }\end{array}$ \\
\hline Surface basale & 60,83 & 35,7 & 57,4 & 53 \\
\hline Richesse floristique & 123 & 42 & 72 & 25 \\
\hline Densité (Tiges/ha) & 692 & 308 & 757 & 200 \\
\hline Shannon-Weaver (H') & 6,94 & 2,93 & 3,02 & 2,08 \\
\hline Simpson (D) & 0,00096 & 0,093 & 0,085 & 0,011 \\
\hline Equitabilité de Piélou (E) & 1,44 & 0,78 & 0,71 & 0,64 \\
\hline Margalef (Rg $\left(\mathrm{R}_{\mathrm{Mg}}\right)$ & 17,54 & 41,83 & 9,69 & 3,98 \\
\hline
\end{tabular}




\section{Usages de quelques essences inventoriées}

Parmi les 51 essences inventoriées sur les deux habitats forestiers, seules quelques-unes recèlent une importance commerciale sur le marché des essences de bois commercial d'Afrique centrale, au nombre desquelles on peut citer : Canarium schweinfurthii, Coelocaryon preussii, Dacryodes pubescens, Erythrophleum suaveolens, Gilbertiodendron dewevrei, Milicia excelsa, Millettia laurentii, Pterocarpus soyauxii, Pycnanthus angolensis, Scorodophloeus zenkeri, Staudtia kamerunensis var. gabonensis et Terminalia superba.

Le Tableau 3 reprenant la classification de (Martin \& Vernay, 2016), mentionne les usages de quelques essences identifiées. Les numéros des espèces sont ceux mentionnés au Tableau 1. Les noms pilotes repris, concernent les idiotaxons observés hors de l'aire échantillonnée.

Tableau 3. Utilisations possibles des essences par type d'ouvrage (Suite)

1. STRUCTURE ET HABILLAGE

\begin{tabular}{|c|c|}
\hline 1.1 Charpente & $50,45,37,38,41,12,19$ \\
\hline 1.2 Lamellé-Collé & $39,37,38,41,12$, \\
\hline 1.3 Carrelet Multi-Plus & Abura, 14, 39, 50, 45, 20, 38, 41, 12,19, \\
\hline 1.4 Contreplaqué Face et contre-face & Abura, 14, 39, 45, Emien, Essessang, 7, 37,20, 38, 41, 19, \\
\hline 1.5 Contreplaqué plis intérieurs & Abura, 14, 49, 26, 50, 45, ESSIA, 42,37, 38, 51, 41, 12, 5, 3, 21, \\
\hline 1.6 Placage tranché &
\end{tabular}

\section{MEUISERIE EXTÉRIEURE (FAÇADE DE BÂTIMENT)}

2.1 Revêtement extérieur, Bardage, Clin

2.2 Menuiserie extérieure : Porte, Portefenêtre, Fenêtre

2.3 Claustra, Brise-soleil

2.4 Fermeture, Volet

$39,26,50,45,42,37,20,11,5,21$

$49,26,50,45,42,37,38,11,5,21$

$26,38,11,3$

$50,45,37,38$

3. MENUISERIE ET AMÉNAGEMENT INTÉRIEUR

\begin{tabular}{|c|c|}
\hline 3.1 Parquet & $26,50,42,37,38,51,12,5,3,19,21$ \\
\hline 3.2 Escalier intérieur & $26,50,45,37,38,41,11,12,5,3$ \\
\hline 3.3 Porte et Huisserie & $\begin{array}{c}\text { Abura, 14, 49, 26, 50, 45, Emien, 37,20,38,51, 41,11,12, 21, } \\
5,3,19\end{array}$ \\
\hline 3.4 Moulure & Abura, 45, Emien, Essessang, 7, 20,51, 41,21 \\
\hline 3.5 Lambris et Habillage intérieur & Abura, $14,26,50,45,37,20,38,51,41,11,12,5,19,21$ \\
\hline 3.6 Agencement-Ameublement & $\begin{array}{c}\text { Abura, 14, 39, 26,50, 45, Emien, Essessang, 7,37, 20,38, } \\
51,41,12,5,19,21\end{array}$ \\
\hline 3.7 Mobilier et Ébénisterie & Abura, $26,50,37,38,12,5,3$ \\
\hline \multicolumn{2}{|c|}{ 4. AMÉNAGEMENT EXTÉRIEUR-LOISIR } \\
\hline 4.1 Escalier extérieur et garde-corps & $26,5,3,36$ \\
\hline $\begin{array}{l}\text { 4.2 Terrasse de plain-pied, Plage de } \\
\text { piscine }\end{array}$ & $49,26,37,38,11,5,3,36$ \\
\hline $\begin{array}{l}\text { 4.3 Terrasse en élévation, Balcon, } \\
\text { Coursive }\end{array}$ & $26,5,3,36$, \\
\hline
\end{tabular}




\begin{tabular}{|c|c|}
\hline $\begin{array}{l}\text { 4.4 Abri, Mobilier à usage extérieur et } \\
\text { aire de jeux }\end{array}$ & $49,26,5,3,36$ \\
\hline 4.5 Portail & $49,26,5,3,36$, \\
\hline $\begin{array}{l}\text { 4.6 Panneaux brise-vue et brise vent, } \\
\text { Pergola }\end{array}$ & $49,26,5,3,36$, \\
\hline \multicolumn{2}{|c|}{ 5. UTILISATION INDUSTRIELLE ET TRAVAUX LOURDS } \\
\hline $\begin{array}{l}\text { 5.1 Travaux hydrauliques en milieu } \\
\text { marin immergé }\end{array}$ & 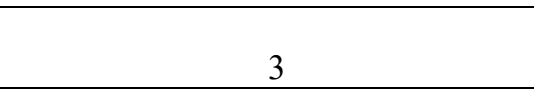 \\
\hline $\begin{array}{l}\text { 5.2 Ouvrage et pont au contact du sol et } \\
\text { de l'eau douce }\end{array}$ & $26,5,3,36$, \\
\hline $\begin{array}{l}\text { 5.3 Ecran acoustique Milieu urbain, } \\
\text { Voies ferrées et routières }\end{array}$ & 26,3 , \\
\hline 5.4 Traverse, Bois de calage & $26,5,3,36$, \\
\hline $\begin{array}{c}\text { 5.5 Plancher industriel et charpente } \\
\text { lourde }\end{array}$ & $39,26,11,5,3,36$ \\
\hline $\begin{array}{l}5.6 \text { Fond de véhicule, Wagon et } \\
\text { conteneur }\end{array}$ & $39,26,38,11,5,3,36$ \\
\hline \multicolumn{2}{|l|}{ 6. CONSTRUCTION NAVALE } \\
\hline 6.1 Bordé et Pont de bateau & $26,38,11,5,3$, \\
\hline \multicolumn{2}{|l|}{ 6.2 Aménagement bateau, Yachting } \\
\hline 6.3 Pontons de plaisance & $26,5,3,36$, \\
\hline \multicolumn{2}{|l|}{ 7. UTLISATIONS DIVERSES } \\
\hline 7.1 Tonnellerie, Cuverie & 38 \\
\hline 7.2 Tournerie, Coutellerie, Brosserie & Abura, $49,50,45,38,41,12,5,3,21$ \\
\hline \multicolumn{2}{|l|}{ 7.3 Manches outil (Bois résilient) } \\
\hline \multicolumn{2}{|l|}{7.4 Instrument de musique } \\
\hline 7.5 Sculpture & Abura, 26, 12, 3, 21, \\
\hline 7.6 Emballage et Caisserie & 14, Abura, 45, 51, 41, 19, \\
\hline
\end{tabular}

Cependant, en raison de leur inscription aux sites Ramsar, ces deux forêts ne sont pas soumises à une exploitation des essences commerciales. Elles ont une vocation de conservation de la biodiversité.

\section{Conclusion}

La présente étude s'est focalisée sur l'inventaire et la répartition des essences de bois commercial sur deux sites forestiers de la ceinture périurbaine de Brazzaville. Cet inventaire complété par le dépouillement des données phytosociologiques et structurales sur ces deux portions écosystémiques, a livré une matrice florale de 51 espèces. Ces dernières s'organisent en 44 genres et 21 familles. Les Fabaceae forment le noyau de ce fond floristique. Les forêts de la Djoumouna et de Loufézou réalisent respectivement une surface basale de 35,7 et $53 \mathrm{~m}^{2} / \mathrm{ha}$. Ramenées à l'échelle spécifique, ce sont Pentaclethra eetveldeana et Pentaclethra macrophylla qui imposent les 
valeurs élevées dans le site de la Djoumouna, alors que sur le site insulaire, ce sont Antiaris toxicaria var. welwitschii et Ceiba pentandra qui se relaient. En ce qui concerne l'indice de valeur d'importance, Pentaclethra eetveldeana et Pentaclethra macrophylla confirment la tendance sur la Djoumouna, alors que Pterocarpus soyauxii et Pentaclethra eetveldeana confortent ce paramètre sur le site insulaire. Ces données viennent compléter la base de données sur la connaissance de la phytodiversité de ces sites particuliers, en vue de l'élaboration des plans d'aménagement à titre conservatoire.

\section{References:}

1. Anonyme (2009). Quatrième Rapport National Sur La Diversité Biologique de la République du Congo. Direction de L'Environnement, Brazzaville, août 2009. 117

2. APG IV (2016). An updated of the Angiosperm Phylogeny Group classifications for orders and families of flowering plants: APG IV (PDF). Botanical Journal of the Linnean Society 181(1) : 1-20. doi $: 10.1111 /$ boj. 12385

3. ATIBT (2016). Nomenclature générale des bois tropicaux. 7e édition Français/Anglais. Association Technique Internationale des Bois Tropicaux, ISBN : 979-10-94410-00-4, 153 p

4. Atlas (2001). Congo. Les Atlas de l'Afrique, éditions Jeune Afrique, Paris : $76 \mathrm{p}$

5. Curtis, J.T. \& McIntosh, R.P. (1951). An upland forest continuum in the prairie-forest border region of Wisconsin. Ecology 32 : 476-496.

6. Dajoz, R. (1996). Précis d'écologie. DUNOD. 551p.

7. Denis, B. (1974). Carte pédologique Brazzaville-Kinkala. Notice explicative $n^{\circ 52}$, République Populaire du Congo à 1.200.000. Paris, ORSTOM.

8. Doucet, J.L. (2003). L'alliance délicate de la gestion forestière et de la biodiversité dans les forêts du Centre du Gabon. Thèse de doctorat, Faculté Universitaire des Sciences Agronomiques de Gembloux, 323 p.

9. Harris, D.J. \& Wortley, A.H. (2008). Les arbres de la Sangha. Manuel d'Identification illustré. Traduction française de Frachon, N. Royal Botanic Garden, Edinburg, United Kingdom: 300 p.

10. Hawthorne, S.W. \& Gyakary, N. (2006). Photoguide for the Forest Trees of Ghana. A treespotter's field guide for indentifying the largest trees. Oxford Forestery Institute, Dpt of Plant Sciences, UK: 432 p. 
11. Hawthorne, S.W. \& Jongkind, C. (2006). Woody plants of western African forest. A guide to the forest trees, shrubs and lianes from Senegal to Ghana. Kew, Roy. Bot. Gardens, Kew: 1023 p.

12. Inoussa, T.M., Imourou, I.T., Gbègbo, M.C. \& Sinsin, B. (2013). Structure et composition floristiques des forêts denses sèches de la région des Monts Kouffé au Bénin. Journal of Applied Biosciences 67 : 4787-4796.

13. Kimpouni, V., Lejoly, J. \& Lisowski, S. (1992). Les Euriocaulaceae du Congo., Frang, Flor.Geobot. 37 (1) $127-145$.

14. Köppen, W. (1936). Das geographische system der klimate. In Köppen, W. et Geiger, R(eds.) Handbuch der klimatologie: 1-44. Berlin.

15. Lebrun, J.-P. \& Stork, A.L. (1991-2015). Enumération des plantes à fleurs d'Afrique

tropicale. Conservatoire et Jardin botaniques de la Ville de Genève, 4 volumes.

http://www.villege.ch/musinfo/bd/cjb/africa/recherche.php?langue=fr

16. Lejoly, J. (1993). Méthodologie pour les inventaires forestiers (Partie flore et végétation). AGRECO-CTFT, Bruxelles, $53 \mathrm{p}$.

17. Louppe, D., Oteng-Amoako, A.A. \& Brink, M. (Editeurs) (2008). Ressources végétales de l'Afrique tropicale 7(1). Bois d'œuvre 1. [Traduction de : Plant Resources ofTropicalAfrica 7(1).Timbers 1.2008].Fondation PROTA,Wageningen, Pays-Bas/ Backhuys Publishers, Leiden, Pays-Bas /CTA,Wageningen, Pays-Bas. 785 pp.

18. Marien, J.N. (2008). Forêt périurbaines et bois énergie : quels enjeux pour l'Afrique

centrale. Rapport sur l'État des forêts d'Afrique centrale : 217-230.

19. Martin, P. \& Vernay, M. (2016). Guide d'utilisation des bois africains écocertifiés en Europe, Tome I. ATIBT, France : 100 p.

20. Mayaux, P., Achard, F. \& Malingreau, J. (1998). Global tropical area measurements derived from coarse resolution imagery: A comparison with other approaches. Environmental Conservation, 25 (1) : 37-52.

21. Meunier, Q., Moumbogou, C. \& Doucet, J.-L. (2015). Les arbres utiles $\mathrm{du}$ Gabon. Presses Agronomiques de Gembloux, $340 \mathrm{p}$.

22. Miabangana, E.S. \& Hondjuila-Miokono, E.D. (2015). Contribution aux études

ethnobotaniques et floristiques de la forêt de la Djoumouna, République du Congo : Les plantes comestibles. Afrique SCIENCE 11(4)

227-240. 
http://www.afriquescience.info/document.php?id=5030. ISSN 1813548X.

23. Miabangana, E.S. \& Hondjuila-Miokono, E.D. (2016). Contribution aux études

ethnobotaniques et floristiques de la forêt de la Djoumouna (République du Congo). Les plantes médicinales, Int. J. Pure App. Biosci. 4(1) : 310-325. doi: http://dx.doi.org/10.18782/23207051.2215

24. Miabangana, E.S. \& Lubini-Ayingweu, C. (2015). Analyse floristique et phytogéographique de la végétation de l'île Loufézou à Brazzaville (République du Congo). Geo-Eco-Trop, 39(1) : 55-66. http://www.geoecotrop.be/uploads/publications/pub_391_03.pdf

25. Miabangana, E.S., Constantin Lubini-Ayingweu, C. \& Malaisse, F. (2016). Analyse floristique et phytogéographique de la forêt de la Djoumouna (République du Congo). Geo-Eco-Trop, 40-2, n.s.: 175190. http://hdl.handle.net/2268/206207

26. Miabangana, E.S. (1998). Etude de quelques plantes utiles de la forêt de la Djoumouna.

Mémoire de fin d'études, option CAPES, Sc.Nat. Université Marien NGouabi, ENS. 48 p., 10 fig. 2 phot.

27. Miabangana, E.S. (2012). Etude floristique, écologique et phytogéographique de

l'écosystème forêt de l'Île Loufézou à Brazzaville (République du Congo). Mémoire de DEA, Sciences de l'Environnement, Fac des Sciences, UNIKIN. 88 p., 6 cartes, 38 fig., 15 table.

28. Miabangana, E.S. (2019). Analyse floristique, phytogéographique et phytosociologique de la végétation insulaire et riveraine du fleuve Congo dans le Plateau des Cataractes (République du Congo). Thèse de doctorat, Université de Kinshasa, Kinshasa : 303 p. + 91 annexes.

29. Miabangana, E.S. (2020). Structure, composition et diversité floristique de la forêt de Djoumouna (République du Congo). European Scientific Journal, April 2020 edition Vol.16. 179-203. https://doi.org/10.19044/esj.2020.v16n12p179

30. Miabangana, E.S. \& Malaisse, F. (2020). Structure, composition et diversité floristiques de l'île forestière Loufézou dans le Plateau des Cataractes (République du Congo). Geo-Eco-Trop., 44, (2): 1-16. http://www.geoecotrop.be/uploads/publications/pub_442_01.pdf 
31. Miabangana, E.S. (inédit). Caractéristiques structurales d'un faciès forestier à Cynometra pedicellata De Wild. \& Pterocarpus soyauxii Taub sur l'île Loufézou dans le Plateau des Cataractes (République du Congo).

32. Mille, G. \& Louppe, D. (coord.) (2015). Mémento du Forestier Tropical. Éditions Quæ, RD 10, 78026 Versailles Cedex, France : 1203 p.

33. Pascal, J.-P. (2003). Notions sur les structures et dynamique des forêts tropicales humides. Rev. For. LV. Numéro spécial, pp. 118-130.

34. Pierlot, R. (1966). Structure et composition de forêts denses d'Afrique centrale, Spécialement celles du Kivu. Ac. Roy. Se. XVI-4, Bruxelles, $367 \mathrm{p}$.

35. Reitsma, J.M. (1988). Forest vegetation of Gabon (végétation forestière du Gabon).

Tropenbos Tech. Sér. 1 : 142 p. Wageningen.

36. Rollet, B. (1983). La régénération naturelle dans les trouées. Bois et Forêts des Tropiques 201, 3-34; 202, 19-33.

37. Samba-Kimbata, M.J. (1978). Le climat Bas-Congolais. Dijon, Université de Dijon, thèse de 3ème cycle, Géographie 280 p., 132 fig., 64 tabl.

38. Tailfer, Y. (1990). La Forêt dense d'Afrique centrale - Identification pratique des principaux arbres. Agence de Coopération Culturelle et Technique et CTA, Wageningen, Tomes 1 (456p) et 2 (1271p).

39. Vande Weghe, J.P., Bidault, E. \& Stévart, T. (2016). Plantes à fleurs du Gabon. Une introduction à la flore des angiospermes. (Col.Marc S.M. Sosef) Agence Nationale des Parcs Nationaux (ANPN), Libreville, Gabon : 792 p.

40. Vivien, J. \& Faure, J.-J. (2011). Arbres des forêts denses d'Afrique centrale. Clohars Carnoet, France, Nguila Kerou, 945 p.

41. White, F. (1986). La végétation de l'Afrique. Mémoire accompagnant la carte de la

végétation de l'Afrique. UNESCO/AETFA/UNSO, ORSTOMUNESCO: $384 \mathrm{p}$

42. White, L. \& Edwards, A. (eds.) (2000). Conservation research in the African rain forests: a technical handbook. Wildlife Conservation Society, New York. 444 p.

43. White, L. \& Vande weghe, J.P. (2008). Patrimoine mondial Naturel d'Afrique centrale : Biens existants - Biens potentiels. Rapport de l'atelier de Brazzaville du 12-14 mars 2008.Unesco - CAWHFI, 98 p. 